\title{
Wide-Angle Intraocular Imaging and Localization ${ }^{\star}$
}

\author{
Christos Bergeles $^{1}$, Kamran Shamaei ${ }^{1}$, Jake J. Abbott ${ }^{1,2}$, \\ and Bradley J. Nelson ${ }^{1}$ \\ 1 Institute of Robotics and Intelligent Systems, ETH Zurich, 8092 Zurich, \\ Switzerland \\ \{cbergeles, kamrans, bnelson\}@ethz.ch \\ 2 Department of Mechanical Engineering, University of Utah, Salt Lake City, \\ Utah 84112 \\ jake.abbott@utah.edu
}

\begin{abstract}
Vitreoretinal surgeries require accuracy and dexterity that is often beyond the capabilities of human surgeons. Untethered robotic devices that can achieve the desired precision have been proposed, and localization information is required for their control. Since the interior of the human eye is externally observable, vision can be used for their localization. In this paper we examine the effects of the human eye optics on imaging and localizing intraocular devices. We propose a method for wide-angle intraocular imaging and localization. We demonstrate accurate localization with experiments in a model eye.
\end{abstract}

\section{Introduction}

Future ophthalmic surgeries will be partially automated in order to achieve the accuracy needed for operations near the sensitive structure of the human retina. Our work is motivated by the untethered intraocular microrobot presented in [1]. The microrobot can move in the posterior of the human eye and is magnetically controlled; for accurate control, knowledge of its position inside the magnetic field is necessary [2]. Since the interior of the human eye is externally observable, vision-based 3D localization can be performed.

Intraocular imaging has been practiced for centuries, and clinicians now have the ability to acquire high-definition, magnified images of the interior of the eye using optical tools that are designed specifically for the human eye. However, these tools combined with the optical elements of the eye (Fig. 1(a)) alter the formation of images (Fig. 1(b)) and make localization challenging.

Our approach to intraocular localization is based on depth-from-focus [3]. Focus-based methods do not require a model of the object of interest, but only knowledge of the optical system. Focus-based methods applied in the eye could

\footnotetext{
* This work was supported by the NCCR Co-Me of the Swiss National Science Foundation. Part of this work was carried out while J. J. Abbott was with the Institute of Robotics and Intelligent Systems.
} 
(a)

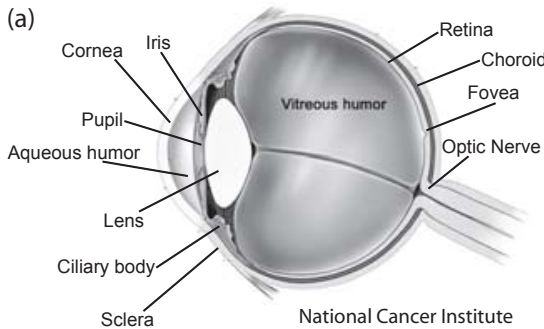

(b)

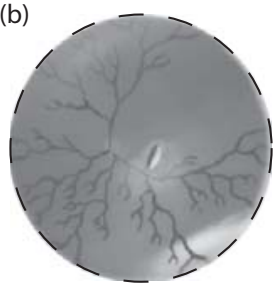

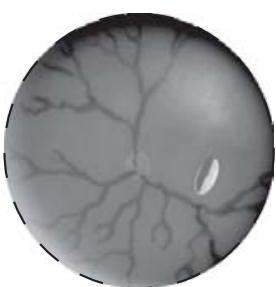

Fig. 1. (a) Anatomy of the human eye. (b) The biomedical microrobot of [1] in the model eye 4. The left image shows the intraocular environment without the eye's optical elements, and the right image shows the effect of the model eye optics. Images are taken with an unmodified digital camera.

also localize unknown objects such as floaters. As a result, our analysis need not be considered only in the scope of microrobot localization, but is applicable on any type of unknown foreign bodies.

In the following section, we consider a stationary camera and evaluate different ophthalmoscopic techniques based on their imaging and localizing capabilities. In Sec. 3 we present a wide-angle localization algorithm. Our experimental results can be found in Sec. 4, and we conclude with an outlook in Sec. 5

\section{Comparison of Ophthalmoscopy Methods}

Our results are based on Navarro's schematic eye [5] (i.e. an optical model based on biometric data that explains the optical properties of the human eye). Navarro's schematic eye performs well for angles up to $70^{\circ}$ measured from the center of the pupil and around the optical axis. For greater angles, the biometric data of each patient should be considered individually. Simulations are carried out with the OSLO optical lens design software. Throughout this section, the object's depth $z$ is measured along the optical axis. We begin by investigating the feasibility of imaging and localizing intraocular devices using existing ophthalmoscopy methods.

\subsection{Direct Ophthalmoscopy}

In a relaxed state, the retina is projected through the eye optics as a virtual image at infinity. An imaging system can capture the parallel beams to create an image of the retina. In direct ophthalmoscopy the rays are brought in focus on the observer's retina [6]. By manipulating the formulas of [7] the field-of-view for direct ophthalmoscopy is found as $10^{\circ}$ (Fig. 2(a)).

Every object inside the eye creates a virtual image. These images approach infinity rapidly as the object approaches the retina. Figure 3 (solid line) displays the distance where the virtual image is formed versus different positions of an intraocular object. In order to capture the virtual images that are created from objects close to the retina, an imaging system with near to infinite working 

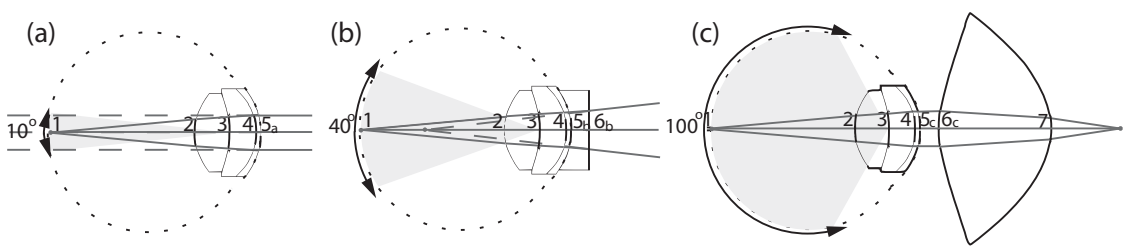

Fig. 2. (a) Direct ophthalmoscopy with Navarro's schematic eye [5]. (b) Ophthalmoscopy with Navarro's schematic eye with a vitrectomy lens 8 . (c) Indirect ophthalmoscopy with Navarro's schematic eye with a condensing lens [9].

Table 1. Optical parameters for the systems of Fig. 2

\begin{tabular}{|r|rrrrrrrrrr|}
\hline Surface & 1 & 2 & 3 & 4 & $5 \mathrm{a}$ & $5 \mathrm{~b}$ & $5 \mathrm{c}$ & $6 \mathrm{~b}$ & $6 \mathrm{c}$ & 7 \\
\hline Radius (mm) & 12.00 & 6.00 & -10.20 & -6.50 & -7.72 & -7.72 & -7.72 & $\infty$ & 11.65 & -9.48 \\
Conic Constant & 0.00 & -1.00 & -3.13 & 0.00 & -0.26 & -0.26 & -0.26 & 0.00 & -9.24 & -1.07 \\
Thickness (mm) & 16.32 & 4.00 & 3.05 & 0.55 & $\infty$ & 2.00 & 2.00 & $\infty$ & 13.00 & $\infty$ \\
Refraction Index & 1.336 & 1.420 & 1.337 & 1.376 & 1.000 & 1.425 & 1.000 & 1.000 & 1.523 & 1.000 \\
\hline
\end{tabular}

distance is required. Such an imaging system will also have a large depth-offield, and depth information from focus would be insensitive to object position.

\section{$2.2 \quad$ Vitrectomy Lenses}

To visualize devices operating in the vitreous humor of phakic (i.e. intact intraocular lens) eyes, only plano-concave lenses (Fig. 2(b)) need to be considered [6]. Vitrectomy lenses cause the virtual images of intraocular objects to form inside the eye, allowing the imaging systems to have a reduced working distance. Based on data given from HUCO Vision SA for the vitrectomy lens S5.7010 [8], we simulated the effects of a plano-concave vitrectomy lens on Navarro's eye (Fig. $2(\mathrm{~b})$ ). This lens allows for a field-of-view of $40^{\circ}$, significantly larger than the one obtainable with the method described in Sec. 2.1

As shown in Fig. 3 (dashed line), the virtual images are formed inside the eye and span a lesser distance. Thus, contrary to direct observation, imaging with an optical microscope (relatively short working distance and depth-of-field) is possible. The working distance of such a system must be at least $20 \mathrm{~mm}$. As depth-of-field is proportional to working distance, there is a fundamental limit to the depth-from-focus resolution achievable with vitrectomy lenses.

\subsection{Indirect Ophthalmoscopy}

Indirect ophthalmoscopy (Fig. 2(c)) allows for a wider field of the retina to be observed. A condensing lens is placed in front of the patient's eye, and catches rays emanating from a large retinal area. These rays are focused after the lens, creating an aerial image of the patient's retina. Condensing lenses compensate for the refractive effects of the eye, and create focused retinal images.

We simulated the effects of a double aspheric condensing lens based on information found in 9 . This lens, when placed $5 \mathrm{~mm}$ from the pupil, allows 


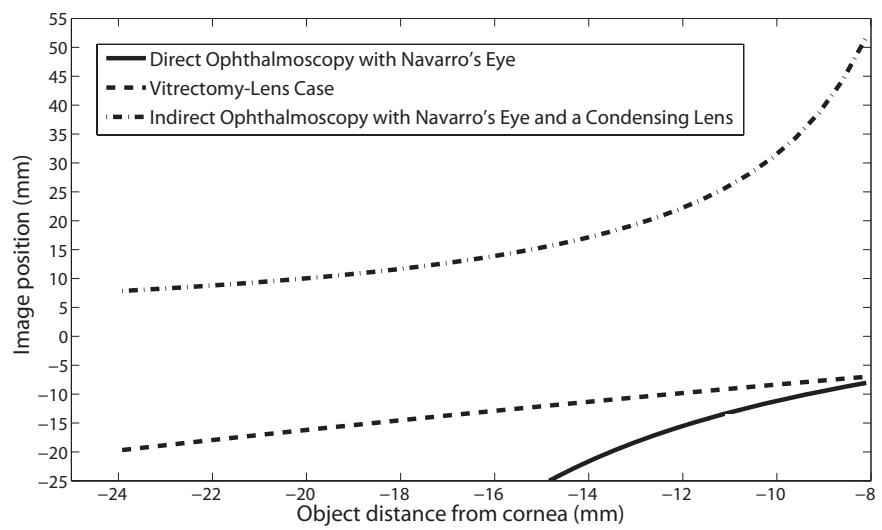

Fig. 3. Image position versus intraocular object position for the direct ophthalmoscopy case, the vitrectomy-lens case, and the indirect ophthalmoscopy case. Image distances are measured from the final surface of each optical system (5a, 6b, 7 respectively).

imaging of the peripheral retina and offers a field-of-view of $100^{\circ}$. As a result, it can be part of an imaging system with a superior field-of-view than the ones described in Sec. 2.1 and Sec. 2.2. The image positions versus the intraocular object positions can be seen in Fig. 3 (dashed-dotted line). A sensing system with a short working-distance and shallow depth-of-field can be used in order to extract depth information from focus for all areas inside the human eye. Depth estimation is more sensitive for objects near the intraocular lens, since smaller object displacements result in larger required focusing motions.

Dense CMOS sensors have a shallow depth-of-focus, and as a result, they can be used effectively in depth-from-focus techniques. Based on Fig. 3 , to localize objects in the posterior of the eye a sensor travel of $10 \mathrm{~mm}$ is necessary. A $24 \times 24 \mathrm{~mm}^{2}$ CMOS sensor can capture the full field-of-view. The simulated condensing lens causes a magnification of $0.78 \times$ and thus, a structure of $100 \mu \mathrm{m}$ on or near the retina will create an image of $78 \mu \mathrm{m}$. Even with no additional magnification, a CMOS sensor with a common sensing element size of $6 \times 6 \mu \mathrm{m}^{2}$ will resolve small retinal structures sufficiently. As a conclusion, direct sensing of the aerial image leads to a high field-of-view, while having advantages in focus-based localization.

\section{Wide-Angle Localization}

As previously stated, the condensing lens projects the spherical surface of the retina onto a flat aerial image. Moving the sensor with respect to the condensing lens focuses the image at different surfaces inside the eye, which we call isofocus surfaces. The locus of intraocular points that are imaged on a single pixel is called an isopixel curve. Figure 4(a) shows a subset of these surfaces and curves and their fits for the system of Fig. 2(c). The position of an intraocular point is found as the intersection of its corresponding isopixel curve and isofocus surface. 

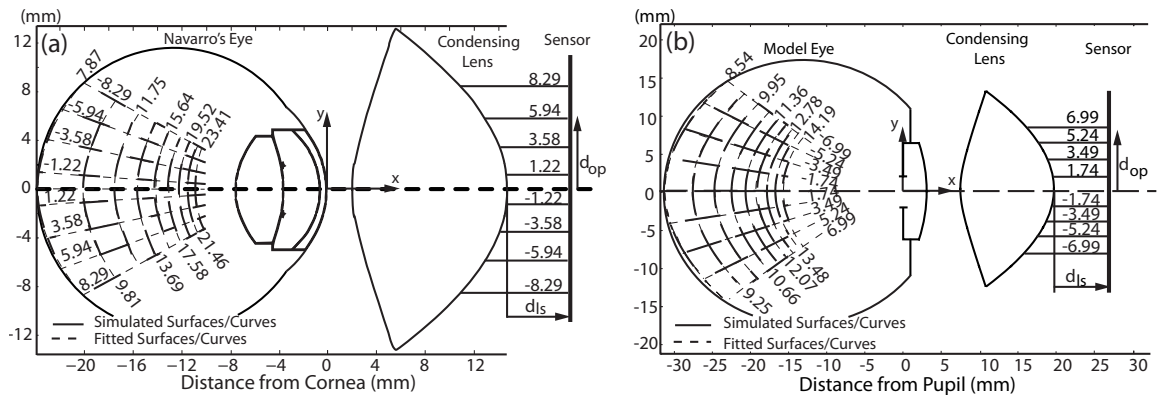

Fig. 4. Simulation of the isofocus surfaces and isopixel curves for (a) indirect ophthalmoscopy with Navarro's eye, and (b) indirect ophthalmoscopy with the model eye 4]. The different isofocus surfaces correspond to the distance from the lens to the sensor $\left(d_{l s}\right)$, for uniform sensor steps of $(\mathrm{a}) \sim 1.95 \mathrm{~mm}$, and $(\mathrm{b}) \sim 0.7 \mathrm{~mm}$. The isopixel curves correspond to pixel distances from the optical axis $\left(d_{o p}\right)$, for uniform steps of (a) $\sim 2.25 \mathrm{~mm}$, and (b) $\sim 1.75 \mathrm{~mm}$.

The location of the isofocus surfaces and isopixel curves are dependent on the condensing lens and the individual eye. The optical elements of the human eye can be biometrically measured. For example, specular reflection techniques or interferometric methods can be used to measure the cornea 10, and autokeratometry or ultrasonometry can be used to measure the intraocular lens [1]. Then, the surfaces and curves can be accurately computed offline using raytracing. In theory there is an infinite number of isofocus surfaces and isopixel curves, but in practice there will be a finite number due to the resolution of sensor movement and pixel size, respectively.

The density of the isofocus surfaces for uniform sensor steps in Fig. 4(a) demonstrates that the expected depth resolution is higher for regions far from the retina. The isopixel curves show that the formed image is inverted, and from their slope it is deduced that the magnification of an intraocular object increases farther from the retina. As a result, we conclude that both spatial and lateral resolutions increase for positions farther from the retina.

The isofocus surfaces result from the optics of a rotationally symmetric and aligned system composed of conic surfaces. We therefore assume that they are conic surfaces as well, which can be parametrized by their conic constant, curvature, and intersection with the optical axis. Since the isofocus surfaces correspond to a specific sensor position, their three parameters can also be expressed as functions of the sensor position.

The isopixel curves are lines, and it is straightforward to parametrize them using their slope and their distance from the optical axis at the pupil. Each isopixel curve corresponds to one pixel on the image, and its parameters are functions of the pixel's offset (measured from the image center) due to the rotational symmetry of the system. For the $2 \mathrm{D}$ case, two parameters are required.

In Fig. 5(a)-(d) the parametrizing functions of the isofocus surfaces and isopixel curves are displayed. The conic constant need not vary (fixed at -0.5 ) because it was observed that the surface variation can be successfully captured 

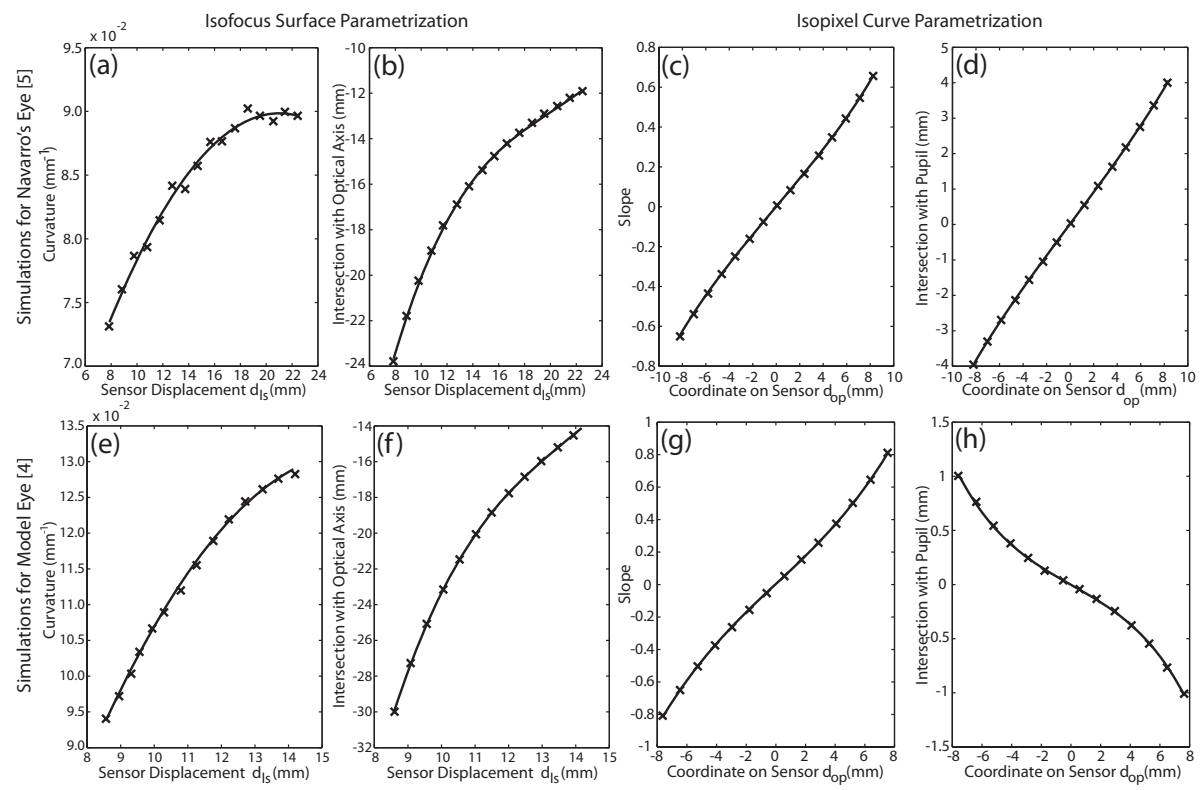

Fig. 5. (top row) Parametrization polynomials for the system of Fig. 4(a), and (bottom row) for the system of Fig. 4(b). Isofocus surface parametrization: (a), (b), (e), (f) Fitted 2nd- and 3rd-order polynomials for the curvature and for the intersection with the optical axis. Isopixel curve parametrization: (c), (d), (g), (h) Fitted 3rd-order polynomials for the line slope and for the intersection with the pupil.

by the curvature. For each parameter, we fit the least-order polynomial that

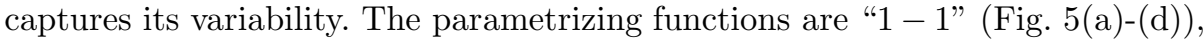
and thus, 3D intraocular localization with a wide-angle is unambiguous.

\section{Localization Experiments}

As an experimental testbed, we use the model eye [4] from Gwb International, Ltd. This eye is equipped with a plano-convex lens of $36 \mathrm{~mm}$ focal length that mimics the compound optical system of the human eye. Gwb International, Ltd. disclosed the lens' parameters so that we can perform our simulations. We also measured the model's retinal depth and shape.

The optical system under examination is composed of this model eye and the condensing lens of Fig. 2(c), where the refraction index was chosen as 1.531. The simulated isofocus surfaces and isopixel curves of the composite system, together with their fits, are shown in Fig. 4(b). Based on these simulations, we parametrize the isofocus surfaces and the isopixel curves (Fig. $5(\mathrm{e})-(\mathrm{h})$ ). The behavior of the parameters is similar to the one displayed in Fig. [5(a)-(d) for Navarro's schematic eye. We assume an invariant conic constant of -1.05 , because the variability of the surfaces can be captured sufficiently by the curvature. 

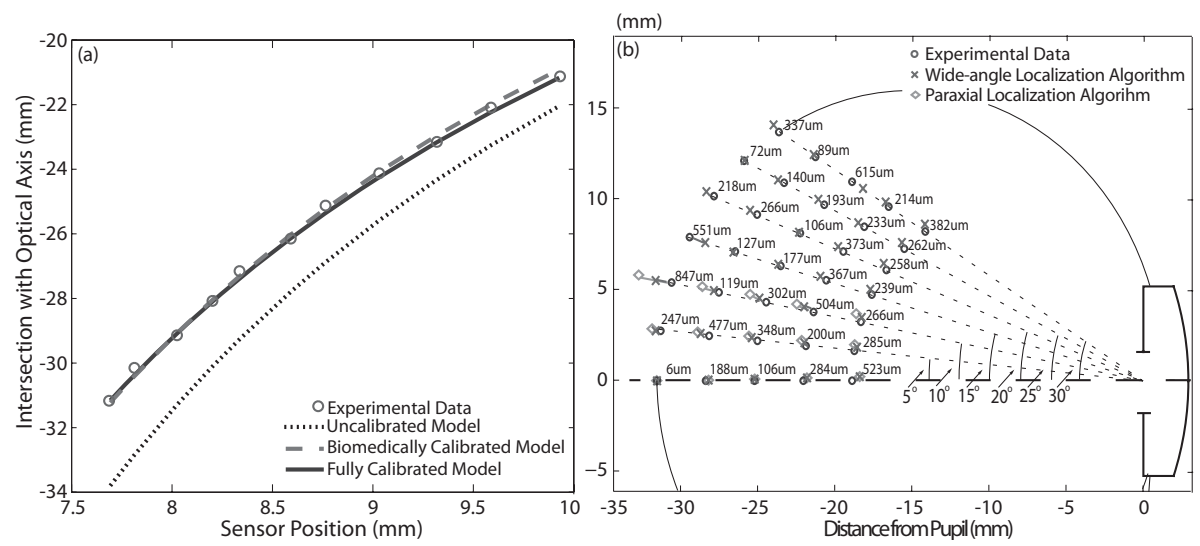

Fig. 6. (a) Model fits for the function describing the intersection of the isofocus surfaces with the optical axis. Biometric calibration errors: mean $=159 \mu \mathrm{m}$, std $=94 \mu \mathrm{m}$. (b) Localization experiment showing the validity of the proposed algorithm. Errors: mean $=282 \mu \mathrm{m}$, std $=173 \mu \mathrm{m}$.

In order to calibrate the isofocus surfaces for their intersection with the optical axis, we perform an on-optical-axis depth-from-focus experiment on the aligned optical system. We use a Sutter linear micromanipulation stage to move a checkerboard calibration pattern in the model eye with $1 \mathrm{~mm}$ steps, and estimate the in-focus sensor position [12. The estimated sensor positions with respect to different object depths can be seen in Fig. 6(a). The uncalibrated model fit is displayed with a solid line, and, as can be seen, calibration is needed.

In the model eye, we can calibrate for the relationship between the in-focus sensor position and the depth of the object using the full set of data points. However, such an approach would be clinically invasive as it would require a vitrectomy and a moving device inside the eye. The only minimally invasive biometric data available are the depth and shape of the retina that can be measured from MRI scans [13. Assuming that there are accumulated errors that can be lumped and included as errors in the estimated image and object positions, it is shown in [14 that by using a first-order model of the optics, calibration using only the depth of the retina is possible. By adapting this method to our framework, we are able to biometrically calibrate for the parameters of the polynomial that describes the intersection of the isofocus surfaces with the optical axis. The resulting fit can be seen in Fig. 6)(a).

The remaining two parameters of the isofocus surfaces control the shape of the isofocus surfaces but not their position. The condensing lens is designed to create a flat aerial image of the retinal surface, and our experiments have shown that we can use it to capture an overall sharp image of the model eye's retina. Therefore, we conclude that there exists an isofocus surface that corresponds to the retinal surface, and we consider it as the $1^{\text {st }}$ surface. From Fig. 4(b) we see that the $1^{\text {st }}$ isofocus surface does indeed roughly correspond to the retinal shape (mean error $=371 \mu \mathrm{m})$. As a result, calibration for the conic constant and the 
curvature is not needed. If our model was not accurately predicting the shape of the retina, then we would calibrate the parameters of the $1^{\text {st }}$ isofocus surface so that is has exactly the same shape as the retina.

To estimate the validity of the presented wide-angle localization algorithm, we consider points in the model eye for various angles with respect to the optical axis and various distances from the pupil. In Fig. 6(b), the results using the proposed wide-angle localization algorithm are displayed. For comparison, we also show the results based on the paraxial localization algorithm presented in 14 for angles up to $10^{\circ}$ from the optical axis. The paraxial localization results deteriorate as the angles increase. However, the localization method proposed here can be used for regions away from the optical axis with high accuracy.

\section{Conclusions and Future Work}

In this paper we evaluated existing ophthalmoscopy techniques based on their imaging and localizing capabilities. We presented the first wide-angle intraocular localization algorithm, and demonstrated accurate localization in a model eye (error: $282 \pm 173 \mu \mathrm{m}$ ). We are currently interested in localizing microrobots $(\sim$ $500 \mu \mathrm{m}$ ) for use in an open-loop magnetic field controller [1, and such localization errors are acceptable. Then, closed-loop visual servoing techniques will be used.

In the future, we will evaluate the robustness of the presented approach with respect to variations in illumination and uncertainties in the optical system. This is an important step towards in vivo intraocular localization.

\section{References}

1. Yesin, K.B., Vollmers, K., Nelson, B.J.: Modeling and control of untethered biomicrorobots in a fluidic environment using electromagnetic fields. Int. J. Robot. Res. 25(5-6), 527-536 (2006)

2. Abbott, J.J., Ergeneman, O., Kummer, M.P., Hirt, A.M., Nelson, B.J.: Modeling magnetic torque and force for controlled manipulation of soft-magnetic bodies. IEEE Trans. Robot. 23(6), 1247-1252 (2007)

3. Ens, J., Lawrence, P.: An investigation of methods for determining depth from focus. IEEE Trans. Pattern Anal. Machine Intell. 15(2), 97-108 (1993)

4. Gwb International, Ltd.: Model eye (2mm pupil) (2009), http://www.gwbinternational.com/model_eye.htm

5. Escudero-Sanz, I., Navarro, R.: Off-axis aberrations of a wide-angle schematic eye model. J. Opt. Soc. A. 16(8), 1881-1891 (1999)

6. Snead, M.P., Rubinstein, M.P., Jacobs, P.M.: The optics of fundus examination. Sur. Ophthalm. 36(6), 439-445 (1992)

7. Smith, G., Atchison, D.A.: The eye and visual optical instruments. Cambridge University Press, Cambridge (1997)

8. FCI Ophthalmics: S5.7010 planoconcave lens (2009), http://www.fci-ophthalmics.com/html/retina.html\#lenses

9. Volk, D.A.: Indirect ophthalmoscopy lens for use with split lamp or other biomicroscope (January 6, 1998) U.S. Patent 5,706,073 
10. Mejia-Barbosa, Y., Malacara-Hernandez, D.: A review of methods for measuring corneal topography. J. Opt. Vis. Sci. 78(4), 240-253 (2001)

11. Kirschkamp, T., Dunne, M., Barry, J.C.: Phakometric measurement of ocular surface radii of curvature, axial separations and alignment in relaxed and accommodated human eyes. Ophthal. Phys. Opt. 24(2), 65 (2004)

12. Sun, Y., Duthaler, S., Nelson, B.J.: Autofocusing in computer microscopy: selecting the optimal focus algorithm. J. Microsc. Res. Tech. 65(3), 139-149 (2004)

13. Atchison, D.A., Pritchard, N., Schmid, K.L., Scott, D.H., Jones, C.E., Pope, J.M.: Shape of the retinal surface in emmetropia and myopia. Invest. Ophthalm. \& Vis. Sci. 46(8), 2698-2707 (2005)

14. Bergeles, C., Shamaei, K., Abbott, J.J., Nelson, B.J.: On imaging and localizing untethered intraocular devices with a stationary camera. In: IEEE Int. Conf. Biomed. Robot. Biomech., pp. 489-494 (2008) 